\title{
A NOTE ON FREQUENCIES OF A BEAM WITH A HEAVY TIP MASS
}

\section{INTRODUCTION}

Turbomachinery blades with shrouds, water tanks, T.V. towers and windmill supporting structures can be approximated to cantilever beams with a heavy mass at the tip for the purpose of analysis of natural vibrations. Such analysis is of practical importance as these beam-like elements are usually subjected to oscillating aerodynamic forces, which, containing all frequency components, can excite the structure at its resonances. Knowledge o the response of the structure in its lower modes of vibration thus is useful from the point of view of stiffening the structure.

Several authors have earlier studied the dynamic behaviour of beams with several bound ary conditions. Young and Felgar [1] tabulated the characteristic functions representing the normal modes of uniform beams, for several boundary conditions. Lee [2] considered a beam hinged at one end by a rotational spring and having a mass attached at the free end with consideration of the effects of rotary inertia and shear on the fundamental frequency. We observed, from Lee's results, that the tip mass inertial effect is more pronounced in the case of a beam with an increasingly rigid end than for one with a hinged end. This motivated us to study the shear and inertial effects due to a concentrated tip mass on both fundamental and higher frequencies of a beam with a perfectly clamped end. Laura et al. [3] determined the first ten natural frequencies of a clamped-free beam with a finite mass at the free end but considered only the translational inertia (shear) term, neglecting the rotary inertia effect Later the analysis was extended to a beam with a rotationally restrained spring element at one end [4].

In this note the frequency equation is derived for a cantilever beam with a heavy mass attached at its free end, with inclusion of both the shear and rotary inertia effects. In this case the second and third derivatives of the displacement functions become discontinuous at the point of attachment of the extra mass. The boundary conditions are accordingly altered to take this into consideration and the frequency equation is then derived in the usual way. Calculated results for the natural frequencies of the first five modes are presented for various mass and moment of inertia ratios.

\section{THEORY}

The differential equation of motion of a uniform beam is

$$
E I \mathrm{~d}^{4} y / \mathrm{d} x^{4}+\rho A \mathrm{~d}^{2} y / \mathrm{d} x^{2}=0 .
$$

The boundary conditions relevant to this problem are,

$$
\begin{gathered}
\text { at } x=0, y(0, t)=0=y^{\prime}(0, t), \\
\text { at } x=l,-E I \frac{\mathrm{d}^{2} y}{\mathrm{~d} x^{2}}(l, t)=J \frac{\mathrm{d}^{3} y}{\mathrm{~d} x \mathrm{~d} t^{2}}(l, t) \text { and } E I \frac{\mathrm{d}^{3} y}{\mathrm{~d} x^{3}}(l, t)=M \frac{\mathrm{d}^{2} y}{\mathrm{~d} t^{2}}(l, t),
\end{gathered}
$$

where $E$ is Young's modulus, $I$ is the second moment of area, $A$ is the area of cross-section, $\rho$ is the mass density, $M$ is the concentrated mass. $M_{b}$ is the mass of the beam, $J$ is the rotary 
inertia of the attached mass, $y$ is the lateral deflection, $x$ is the distance along the beam from the fixed end and $t$ is the time.

The solution of equation (1) is of the form

$$
y(x, t)=Y(x) \sin \omega t,
$$

where $Y(x)$ is the normal mode of vibration and $\omega$ is the corresponding natural frequency ( $\mathrm{rad} / \mathrm{s})$. The normal mode can be expressed as

$$
Y(x)=C_{1} \cos k x+C_{2} \sin k x+C_{3} \cosh k x+C_{4} \sinh k x,
$$

where

$$
k^{4}=\omega^{2}(\rho A / E I)
$$

and $C_{1}, C_{2}, C_{3}$ and $C_{4}$ are constants.

Substituting equation (4) in equation (3) and then applying the boundary conditions (2) yields a set of homogeneous simultaneous equations in $C_{1}, C_{2}, C_{3}$ and $C_{4}$. The condition that the determinant of the coefficient matrix of these equations vanishes gives the following frequency equation:

$$
\begin{gathered}
\left(J / M_{b} L^{2}\right)\left(M / M_{b}\right) y_{i}^{4}\left(1-\cos y_{i} \cosh y_{i}\right)-\left(J / M_{b} L^{2}\right) y_{i}^{3}\left(\cos y_{i} \sinh y_{i}+\sin y_{i} \cosh y_{i}\right) \\
+\left(M / M_{b}\right) y_{i}\left(\cos y_{i} \sinh y_{i}-\sin y_{i} \cosh y_{i}\right)+\cos y_{i} \cosh y_{i}+1=0,
\end{gathered}
$$

where $y_{i}=k_{i} L, i=1,2,3, \ldots$ being the mode number.

When $J=0$, equation (6) becomes

$$
\left(M / M_{b}\right) y_{i}\left(\cos y_{i} \sinh y_{i}-\sin y_{i} \cosh y_{i}\right)+\cos y_{i} \cosh y_{i}+1=0,
$$

which agrees with the expression derived in reference [3].

When $M$ is zero the equation becomes $\cos y_{i} \cosh y_{i}+1=0$, which is the frequency equation for a uniform cantilever beam.

\section{IMPLEMENTATION OF THE CALCULATION PROCEDURE ON A DCM MICROSYSTEM COMPUTER}

A program has been written for the solution of the frequency equation on a computer. Initial values of the approximate roots, if known, can be set, or the program finds the roots if the tolerance desired in the root is given. The program has been used on a DCM Microsystem computer. To save computer time the procedure of first conducting a search to find approximate roots was used. These approximate roots were later refined to the desired accuracy.

\section{NUMERICAL RESULTS AND DISCUSSION}

The frequency equation (5) was solved for the first five roots on the DCM Microsystem by a direct search procedure to arrive at the approximate roots. Then these roots were refined by halving the interval. The roots for different values of $M / M_{b}=0.0,0.2,0.4,0.6$, 0.8 and 1.0 and $J / M_{b} L^{2}=0.0,0 \cdot 2,0.4,0.6,0.8$ and 1.0 were computed and the results are presented in Table 1 . The values with asterisks need to be further refined, due to the large magnitude the equation attains for a small increase in the increment in the root. For $M / M_{b}=$ 0, the frequency values coincide with the results of Laura et al. [3].

THe results presented in Table 1 show that the presence of a concentrated end mass lowers all the frequencies, although its effect is more pronounced for the fundamental requency than for higher frequencies. The results also indicate the relative importance of the two terms $J / M_{b} L^{2}$ and $M / M_{b}$ to the frequencies. It is obvious from our results that the rotary inertia due to the concentrated mass is more important than the translational in- 
TABLE 1

Values of the first five roots of the frequency equation for ranges of values of the mass and moment of inertia ratios

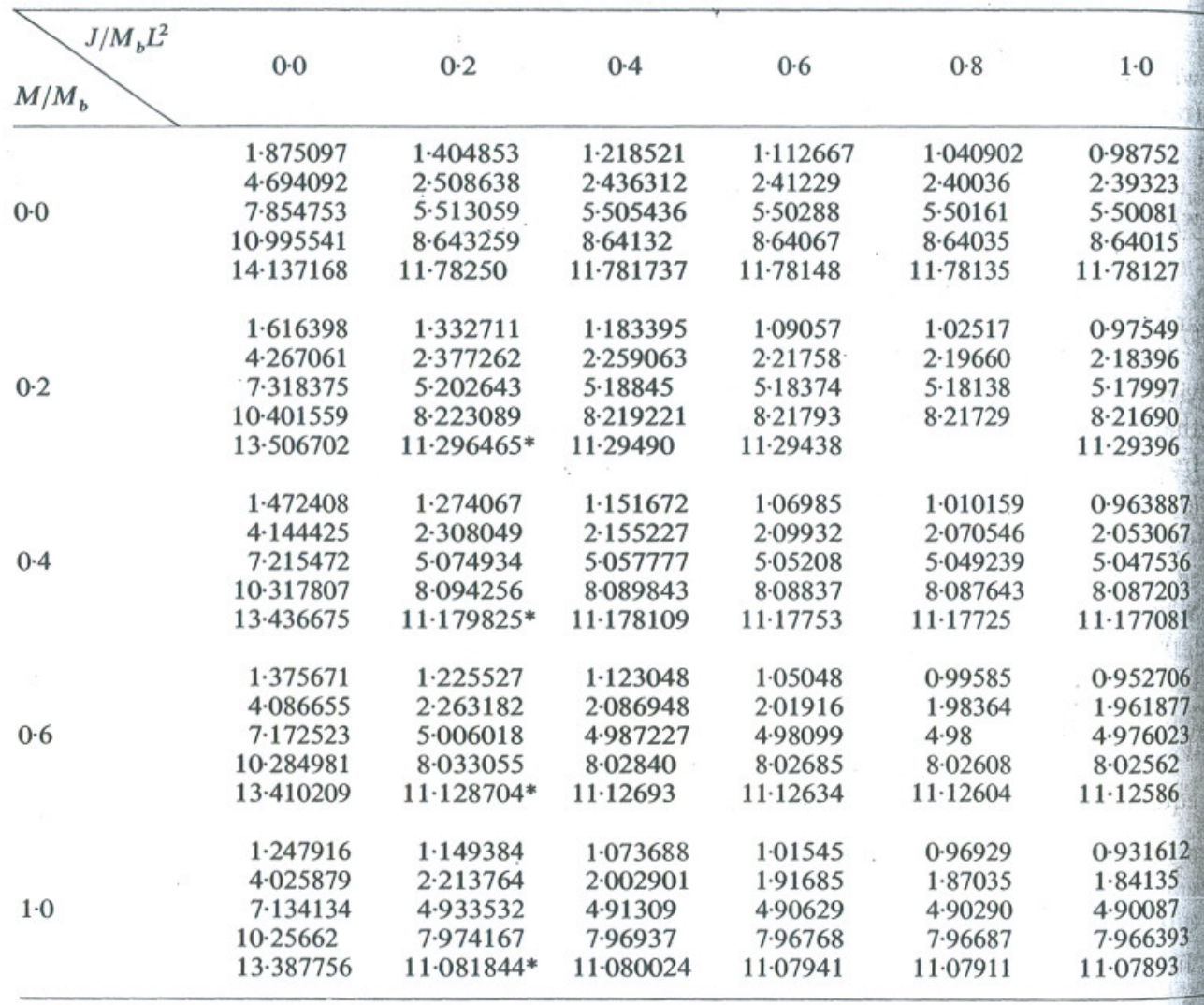

ertia, or shear effect. To cite an example, the first root drops from 1.87509 to 0.9875 for a change in $J / M_{b} L^{2}$ from 0 to 1 whereas the same root changes from 1.87509 to 1.2479 for the same amount of change in $M / M_{b}$. In the case of the fifth root the change is from 14.137 to 11.781 for $J / M_{b} L^{2}$ from 0 to 1 whereas it is 14.137 to 13.387 for an equal change in $M / M_{b}$.

Another interesting observation is that these second order effects due to a concentrated tip mass are more apparent in the case of a beam with a perfectly clamped end than fora beam with a hinged end. This fact is also evident from the results of Lee [2]. For a more rigidly clamped end, for example, for $k L / E I=100$, the first root changes from 1.856 to 0.984 to 0.315 as $J / M_{b} L^{2}$ changes from 0 to 1 to 100 . But for a soft end, i.e., for $k L / E I=0.0$, this change is only from 0.415 to 0.293 to 0.099 . According to our results, a beam with a perfectly clamped end will change its fundamental frequency root from 1.875 to 0.987 as $J / M_{b} L^{2}$ changes from 0 to 1 . No comparison can be made with the results given in refer ence [3] results as for these the rotary inertia term was not considered. The fifth root drops from 14.137 to 11.781 for an equal change in $J / M_{b} L^{2}$. To illustrate the combined effect 0 $J / M_{b} L^{2}$ and $M / M_{b}$ for both a beam with a hinged end and one with a fixed end, first conside a beam with a hinged end, with a flexibility of $k L / E I=1$ as defined by Lee [2] or $E I / L=$ 
as defined by Laura et al. [4]. The first root decreases from $1 \cdot 247$ to $0 \cdot 809$ for $J / M_{b} L^{2}=0$ to 1 in the case of Lee's formulation [2], but it is only from 1.2479 to 0.8704 in the case of Laura et al. [4]. The discrepancy is due to the fact that Laura did not include the rotary inertia effect. In the case of a perfectly or near perfectly clamped end $(\approx k L / E I=100)$, for $J / M_{b} L^{2}=M / M_{b}=1$, the fundamental root of the frequency equation decreases from 1.856 to 0.927 in Lee's case [2], but in the present analysis it changes from 1.875 to $0-931$. The change of the fifth root in the present analysis is from 14.137 to 11.079.

It thus has been shown by the present and previous analyses that the rotary and translational inertia effects have a more pronounced effect on the frequencies of a rigidly clamped beam with a tip mass than on those of a beam with a flexible end. Also the rotary inertia of a concentrated mass is more important than the translational inertia (shear effert) and these corrections must necessarily be considered together for accurate evaluation of natural frequencies of beams with tip masses.

\section{ACKNOWLEDGMENT}

The authors are grateful to Mrs S. Usha, Propulsion Division, for her efforts in carrying out the calculations.

Propulsion Division,

National Aeronautical Laboratory, Bangalore-560037, India

M. SWAMinadHAM

A. Michael

(Received 9 May 1979)

\section{REFERENCES}

D. Young and R. P. Felgar, JR 1949 University of Texas Publication, Austin, Texas, No. 4913. Tables of characteristic functions representing normal modes of vibration of a beam.

2. T. W. LEE 1973 Transactions of the American Society of Mechanical Engineers, Journal of Applied Mechanics 7, 813-815. Vibration frequencies for a uniform beam with one end spring hinged and carrying a mass at the other free end.

3. P. A. A. Laura, J. L. Pombo and E. A. Susemint 1974 Journal of Sound and Vibration 37, 161-168. A note on the vibrations of a clamped-free beam with a mass at the free end.

P. A. A. Laura, M. J. Maurizi and J. L. Pombo 1975 Journal of Sound and Vibration 41, 397-405. A note on the dynamic analysis of an elastically restrained-free beam with a mass at the free end. 